\title{
Quantitative Evaluation of Stroke Patients' Wrist Paralysis by Estimation of Kinematic Coefficients and Machine Learning
}

\author{
Jihun Kim, Wookhyun Park, and Jaehyo Kim* \\ School of Mechanical and Control Engineering, Handong Global University, \\ 558, Handong-ro, Heunghae-eup, Buk-gu, Pohang-si, Gyeongsangbuk-do, Republic of Korea
}

(Received September 20, 2019; accepted November 25, 2019)

Keywords: stroke, hemiplegia, rehabilitation, robotic therapy, machine learning

The increasing population of stroke survivors naturally produces needs for more effective rehabilitation systems for both patients and therapists. Robotic therapies are widely studied and practiced in various fields since they enable intense exercise as well as numerical evaluations. In this paper, along with the rehabilitation robot we developed, we propose a quantitative evaluation method for wrist paralysis in stroke patients using kinematic coefficients estimated from the joint model and machine learning. Through experiments on five hemiplegic patients, we observed the spring-damper characteristics of their paralyzed wrists and computed the coefficients that represent stiffness and viscosity. During wrist extension, a patient at Brunnstrom stage 3 showed a high average stiffness of $4.453 \mathrm{Nm} / \mathrm{rad}$ and viscosity of 4.533 $\mathrm{Nms} / \mathrm{rad}$ toward the rest position, whereas a patient at Brunnstrom stage 4 showed smaller coefficients of $1.135 \mathrm{Nm} / \mathrm{rad}$ and $-0.669 \mathrm{Nms} / \mathrm{rad}$, respectively. We applied a support vector machine and a k-means method to the estimated stiffnesses and viscosities to classify the patients into three different clusters. The two coefficients not only helped discriminate patients in accordance with their Brunnstrom stage, but also revealed that patients at the same stage could be more finely categorized.

\section{Introduction}

A stroke occurs when brain cells are damaged owing to a lack of oxygen caused by either a blocked or ruptured blood vessel in the brain. Although the death rate has been gradually declining since the 1990 s owing to advancements in medical treatments, the prevalence rate is increasing mainly because of unhealthy diets or low physical activity. ${ }^{(1)}$ As a result, the population of stroke survivors naturally and inevitably grows. According to worldwide statistics of early 2010, about 15 million people suffer a stroke every year and two-thirds of them survive. ${ }^{(1,2)}$ The loss of motor functions after brain injury makes the activities of daily living difficult for the survivors. More than $45 \%$ of the survivors experience semipermanent disabilities, including musculoskeletal paralysis. ${ }^{(1,3)}$ To increase the quality of life, months or years of rehabilitation is necessary. ${ }^{(4-6)}$

*Corresponding author: e-mail: jhkim@handong.edu 
Repetitive joint movements and muscle stretches are carried out in hospitals to stimulate neuroplasticity so that neighboring normal neurons can take over the lost motor functions of damaged neurons. ${ }^{(7-9)}$ Conventional treatments involve the application of external forces on a patient's paralyzed joints by a therapist to help improve the patient's muscle tone. However, such treatments are constantly pointed out as being unsatisfactory because the patient's participation is passive and the amount of quantitative data on the results of daily exercise is sparse. ${ }^{(10,11)}$ As a solution to the first limitation, we proposed the robot-assisted bilateral wrist exercise shown in Fig. 1(a) in our previous research, in which the advantages of intense exercise in robotic therapy and the active involvement of patients in mirror therapy are combined. ${ }^{(12)}$ In this paper, as a solution to the second limitation, we suggest a quantitative evaluation of the wrist paralysis of stroke patients by applying kinematic coefficients estimated using the springdamper joint model and the robot sensor readings. Research studies have been conducted to estimate the stiffness and viscosity of paralyzed joints such as ankles, arms, or wrists using personalized rehabilitation robots. ${ }^{(13-16)}$ However, the results seem to differ from paper to paper and are not in agreement with the results of conventional evaluations by doctors and therapists. Thus, for our estimated coefficients, we applied both supervised and unsupervised machine learning methods and compared the results with the subjects' Brunnstrom stages to check whether our results concur with the conventional stroke assessment.

\section{Materials and Methods}

\subsection{Mirroring robot}

The mirroring robot proposed in our previous study is shown in Fig. 1(a). A worm-geared DC motor with a rated torque of $4.41 \mathrm{Nm}$ was chosen to guide the stroke patient's wrist flexion and extension exercises. To monitor such exercises, a torque sensor of $49.03 \mathrm{Nm}$ capacity and a

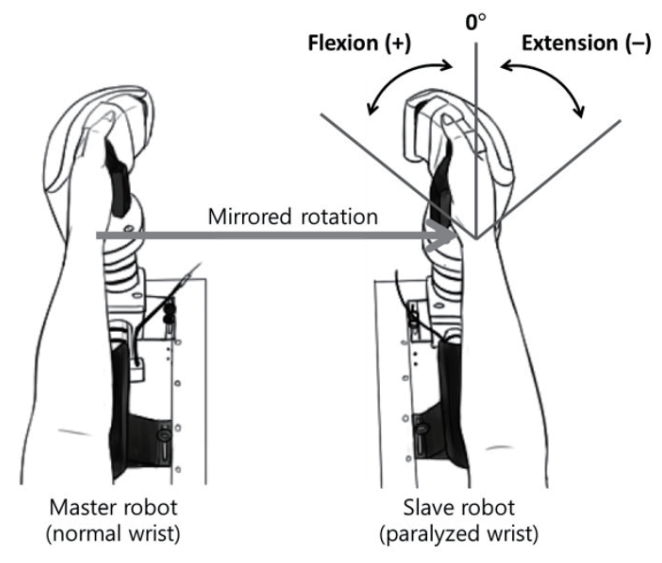

(a)

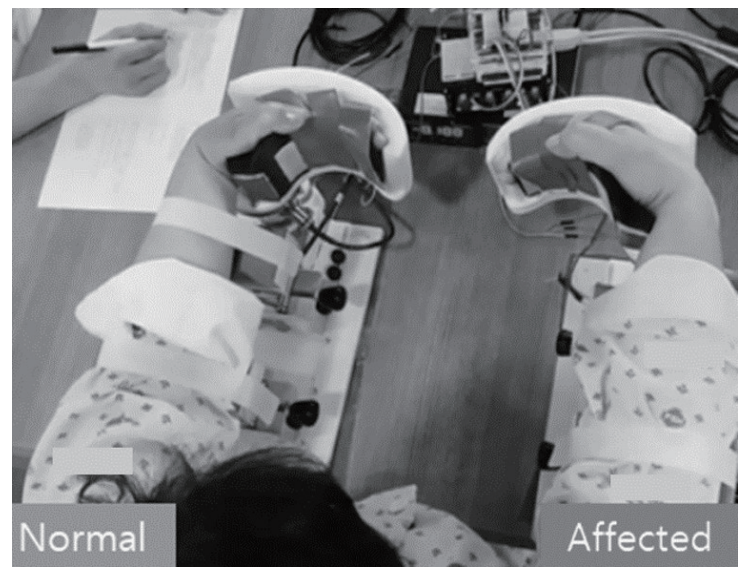

(b)

Fig. 1. Experimental setup with the wrist mirroring robot. (a) Paired set of robots with a 1-DOF range of motion and (b) wrist mirroring exercise of a representative patient. 
position sensor were coupled with the motor. The hand support and handle were customized to the shape of each user's hand and fabricated by 3D printing. A robot was provided to each side to induce bilateral wrist exercises at the user's will. The robot on the normal side can be freely rotated by the user's voluntary wrist movement toward either the flexion or extension direction. Then, the robot on the paralyzed side rotates to an angular position symmetric to that of the normal side to produce the same flexion or extension exercise.

\subsection{Experimental setup}

Five hemiplegic patients at Brunnstrom stages between 3 and 5 participated in this study, as listed in Table 1. All the participants were informed of the purpose and procedure of the study in advance and their written consent was obtained. The protocol was carried out in accordance with the ethical standards of the Declaration of Helsinki. The participants were asked to perform the wrist flexion-extension exercise using the mirroring robot for $10 \mathrm{~min}$, as shown in Fig. 1(b). Using the robot, we measured the torques and angular positions of the patient's paralyzed wrist throughout the exercise. Data were collected using NI-6009 at a sampling rate of $200 \mathrm{~Hz}$ and digitally processed with a 3rd-order Butterworth low-pass filter with a cutoff frequency of $5 \mathrm{~Hz}$, using MALTAB 2019a.

\subsection{Data processing}

After the exercise, we calculated damping $(B)$ and spring constants $(K)$ of the subject's paralyzed wrist, using the following simplified wrist joint kinematic model. ${ }^{(17)}$

$$
-\boldsymbol{\tau} \approx[\boldsymbol{\omega} \theta]\left[B B^{\mathrm{T}}\right.
$$

Since the torque, angular velocity, and angular position vectors are known from the sensor measurements, the kinematic coefficients can be estimated by the least squares method. We have included the negative sign on the torque to reflect its reflexive characteristics against the joint movement.

After the linear regression, we applied a support vector machine (SVM) for supervised machine learning and a k-means method for unsupervised machine learning to categorize the subjects into three classes on the basis of the two parameters. For the SVM, we used the 'fitcsvm' function in MATLAB 2019a. After standardization, each class was linearly separated from the others using a radial basis function $(\mathrm{RBF})$ as a kernel function. We coded a k-means

Table 1

Patient information.

\begin{tabular}{lcccc}
\hline Patient & Age & Sex & Brunnstrom stage & Affected side \\
\hline 1 & 63 & Female & 4 & Left \\
2 & 61 & Female & 4 & Left \\
3 & 66 & Female & 3 & Right \\
4 & 59 & Female & 5 & Right \\
5 & 55 & Female & 3 & Right \\
\hline
\end{tabular}


function ourselves in MATLAB 2019a since the algorithm is very simple compared with that of the SVM. The process of calculating the centers of three clusters and regrouping nearest data points into newly defined clusters was repeated 10000 times until the machine learning reached convergence.

\section{Results}

Figure 2 illustrates two representative patients' wrist positions and torques against time during the flexion-extension exercise. We observed that the positions and torques are completely out of phase. Therefore, we inferred that patients' wrists show reflexive torques against robot rotations toward the rest positions as if the joints were spring-damper systems. The magnitudes observed for the subjects at Brunnstrom stage 3 [Fig. 2(a)] were higher than those observed for the subjects at Brunnstrom stages 4 and 5 [Fig. 2(b)], as expected, since their wrists and fingers had more severe paralysis. In addition, greater reflexive torques were measured, particularly during wrist extension than during flexion, because musculoskeletal contracture is a common symptom of hemiparesis after a stroke. Thus, we proceeded to compute spring and damping constants from the joint kinematic model only during each extension exercise to obtain a clearer estimation.

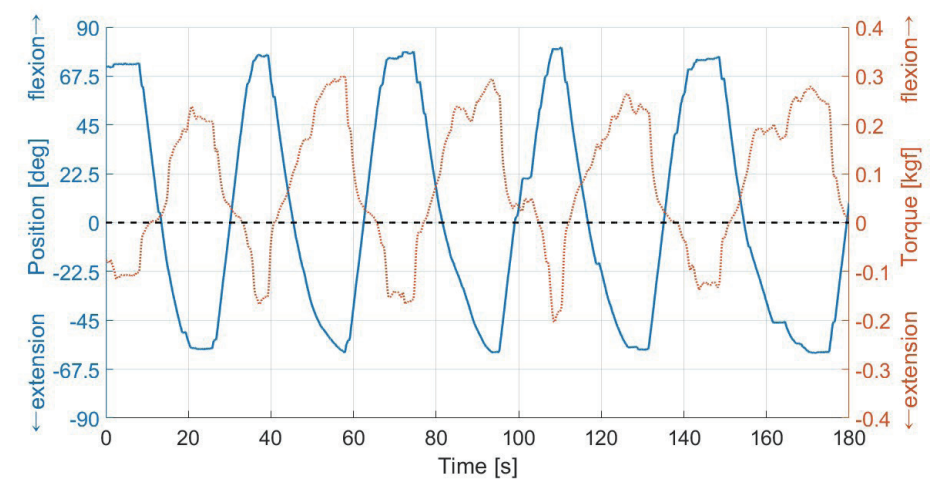

(a)

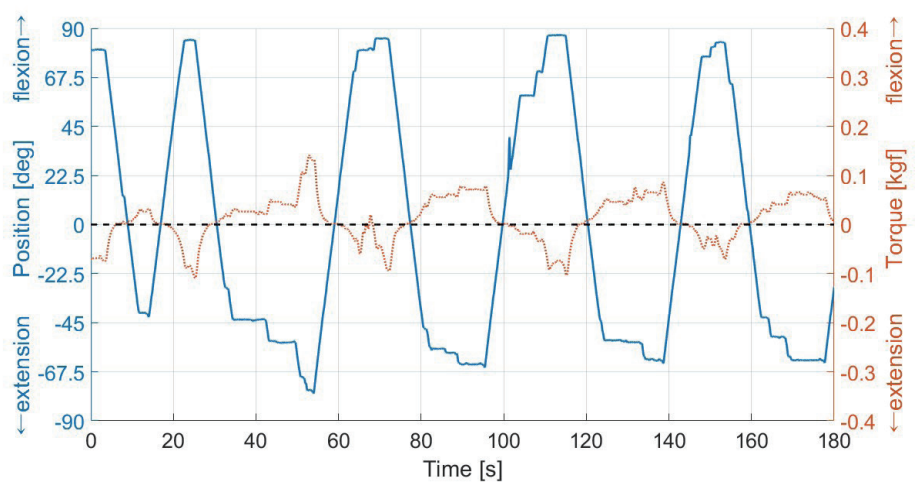

(b)

Fig. 2. (Color online) Wrist position and torque during flexion-extension exercise depicted from 0 to $180 \mathrm{~s}$ out of 10 min. (a) Patient at Brunnstrom stage 3 and (b) patient at Brunnstrom stage 4. 


\subsection{Estimation of kinematic parameters using wrist joint model}

During the 10 min exercise, each patient performed more than 15 sets of wrist flexion and extension. After discarding data with discontinuities or too many fluctuations, we collected measurements of at least 12 wrist extensions from each patient. During extension, we computed the damping constant $B$ and spring constant $K$ by applying the least squares method to the wrist joint model [Eq. (1)], since the reflexive torque, angular position, and angular velocity vectors are known through the robot sensors.

$$
[B K]^{\mathrm{T}}=\left(\left[\begin{array}{ll}
\boldsymbol{\omega} & \boldsymbol{\theta}
\end{array}\right]^{\mathrm{T}}[\boldsymbol{\omega} \boldsymbol{\theta}]\right)^{-1}[\boldsymbol{\omega} \boldsymbol{\theta}]^{\mathrm{T}}(-\boldsymbol{\tau})
$$

After the estimation of the coefficients, by multiplying them with the state vectors, an estimate of the torque vector was calculated to determine its correlation with the actual torque vector. The results in Fig. 3 suggest the validity of $B$ and $K$, since the estimated torque reflects the actual torque well with a high correlation of more than 0.9 .

Table 2 summarizes each patient's average $B$ and $K$ and the Brunnstrom stage provided by physical therapists. First, we observed that the average correlations are quite high with a minimum value of 0.810 . Also, note that the magnitudes of $B$ and $K$ are in accordance with the Brunnstrom stage. In other words, subjects at stage 3 with severe paralysis show greater $B$ and $K$, suggesting that these kinematic parameters are valid indices for describing the wrist condition of hemiplegic patients.

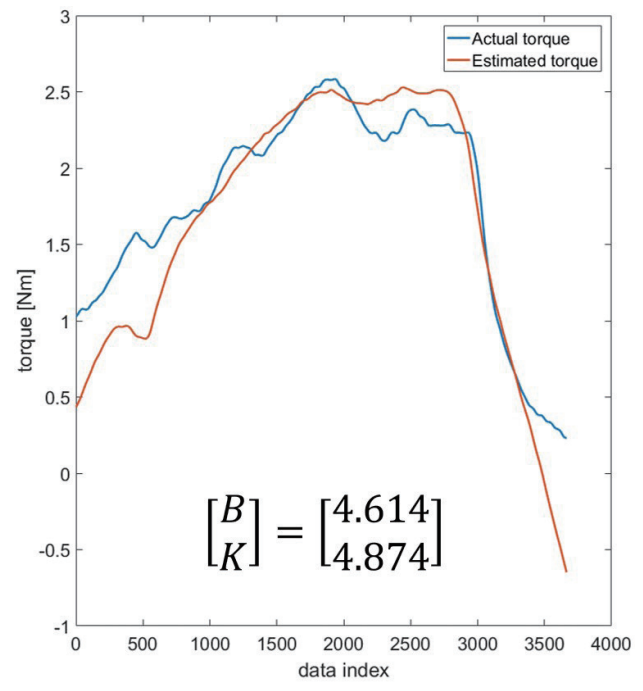

(a)

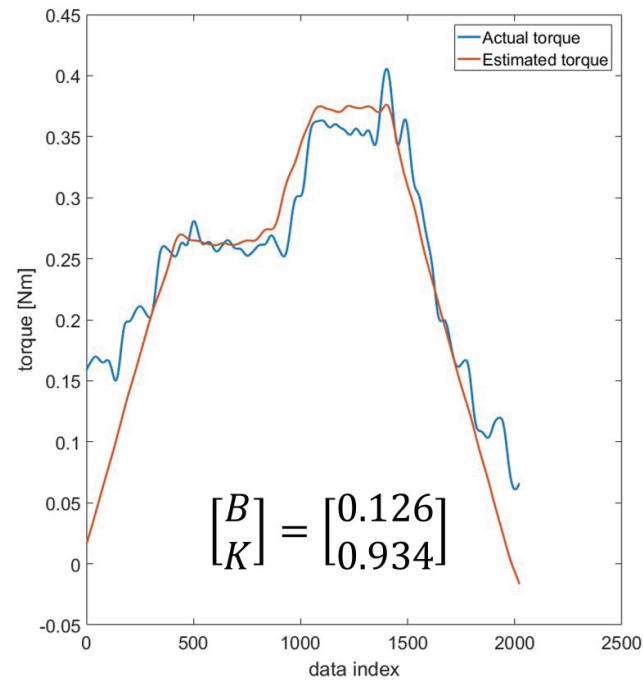

(b)

Fig. 3. (Color online) Estimations of kinematic coefficients during wrist extension exercise. The signs are negative because in Fig. 2 we had set the signs of the reflexive torque vector $\boldsymbol{\tau}$ and state vectors $\boldsymbol{\omega}$ and $\boldsymbol{\theta}$ opposite to each other. During wrist extension, angular positions are negative whereas torques are positive to be reflexive. (a) High viscosity and stiffness with a correlation of 0.970 shown by a patient at Brunnstrom stage 3 and (b) relatively low viscosity and stiffness with correlation of 0.960 shown by a patient at Brunnstrom stage 4 . 
Table 2

Average kinematic coefficients and correlations between actual and estimated torques.

\begin{tabular}{lccccc}
\hline Patient & Brunnstrom stage & Extension sets & $\begin{array}{c}\text { Viscosity, } B \\
(\mathrm{Nms} / \mathrm{rad})\end{array}$ & $\begin{array}{c}\text { Stiffness, } K \\
(\mathrm{Nm} / \mathrm{rad})\end{array}$ & Correlation \\
\hline 1 & 4 & 19 & -0.669 & 1.135 & 0.851 \\
2 & 4 & 22 & -7.872 & 1.846 & 0.834 \\
3 & 3 & 12 & 4.533 & 4.453 & 0.925 \\
4 & 5 & 16 & -9.582 & 0.717 & 0.810 \\
5 & 3 & 16 & 0.866 & 2.024 & 0.910 \\
\hline
\end{tabular}

\subsection{Pattern recognition for quantitative analysis of wrist paralysis}

The kinematic parameters of patients during each extension exercise are plotted on the $B-$ $K$ plane, as shown in Fig. 4. Using the SVM, we classified the patients in accordance with their Brunnstrom stage. Patients at Brunnstrom stage 3, thus in region 1 in Fig. 4, tend to have higher stiffness and viscosity, as expected. However, we observe that the boundaries between the consecutive Brunnstrom stages are unclear because there are some overlapping data points. Furthermore, there exist wide gaps between data points even within the same Brunnstrom stage.

Using k-means, an unsupervised classifier, we identified the severity of wrist paralysis of patients on the basis of only their $B \mathrm{~s}$ and $K \mathrm{~s}$ regardless of their Brunnstrom stage. By setting the cluster size as 3, we get Fig. 5, which shows a different result from Fig. 4. The boundaries are now placed in the middle of the wide gaps, thus merging patients with different Brunnstrom stages. We rated each cluster "severe", "moderate", or "light" in accordance with the magnitudes of stiffness and viscosity. From Fig. 5, we discovered that the severity of wrist paralysis may vary within the same Brunnstrom stage.

\section{Discussion}

In this paper, we proposed a quantitative method for analyzing wrist paralysis in hemiplegic patients. Using torque and angular position vectors measured during the robot-assisted wrist

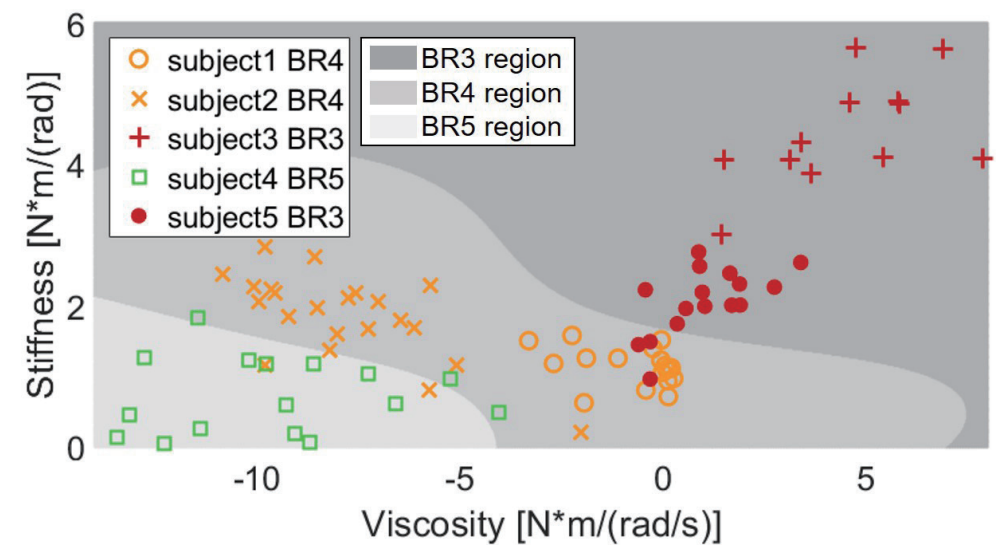

Fig. 4. (Color online) Borders between Brunnstrom stages on the $B-K$ plane discriminated using the SVM. 


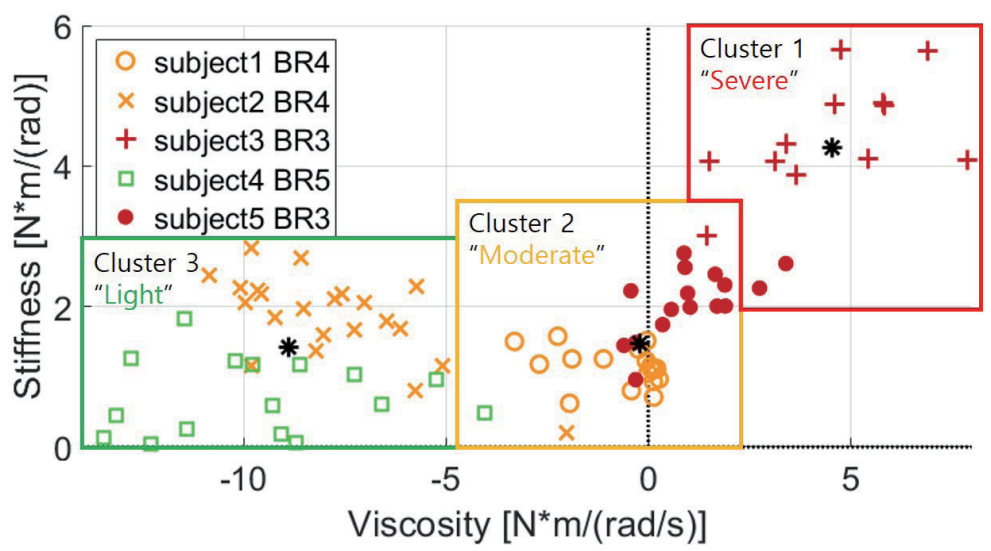

Fig. 5. (Color online) Severity of wrist paralysis quantitatively redefined on the $B-K$ plane using k-means.

extension exercise, we computed the kinematic coefficients — viscosity $B$ and stiffness $K$ - using the simplified joint model. The parameters were then plotted on the $B-K$ plane to perform pattern recognition and redefine the severity of wrist paralysis.

Since we only had data from five hemiplegic patients, the validity of the results is quite weak. Our robot is currently under review for medical licensing, after which it will be installed at a local hospital to collect more patient data. A study using a larger number of data will confirm the reliability of our quantitative evaluation through the use of many machine learning methods other than the SVM method or K-means method, such as the use of an artificial neural network. Once our assessment can provide more reasonable results, therapists and doctors will be able to utilize it as a more specific rehabilitation guide together with existing qualitative indicators including the Brunnstrom, Fugl-Meyer, or Jepsen assessment scale. In addition, long-term studies will show how these parameters change as patients rehabilitate over time. Hopefully, the indices can be used as navigation parameters.

In this paper, we have used the word 'paralysis' to refer to both stiffness $K$ (springlike, position-dependent) and viscosity $B$ (dampinglike, speed-dependent) of the wrist. In the field of rehabilitation, we noticed, after several meetings with therapists and doctors, that terms such as 'muscle tone' or 'spasticity' are frequently used to indicate the patient's joint paralysis to emphasize musculoskeletal viscosity. Therefore, we realized that doctors and therapists are more concerned with the speed-dependent characteristics of a paralyzed joint. Moreover, from Figs. 4 and 5, we see that our optimal estimation method - the least squares methodhas a limitation, since the viscosity data of each subject is relatively widespread. In order to decrease the deviation of viscosity, other estimation methods, such as the use of the Kalman filter, can be chosen. Such methods would increase the low correlations under 0.9 in Table 2. As an alternative solution, we could try surface electromyography of the participants to estimate stiffness and viscosity on the basis of muscle activity. In this case, a more detailed definition of musculoskeletal paralysis would be possible.

For us, the change in the sign of viscosity $B$ was a subject of vigorous debate because a physical constant with a negative sign is unreal in natural mechanical systems. However, the fact that the change in the sign appeared between Brunnstrom stages 3 and 4 caught our 


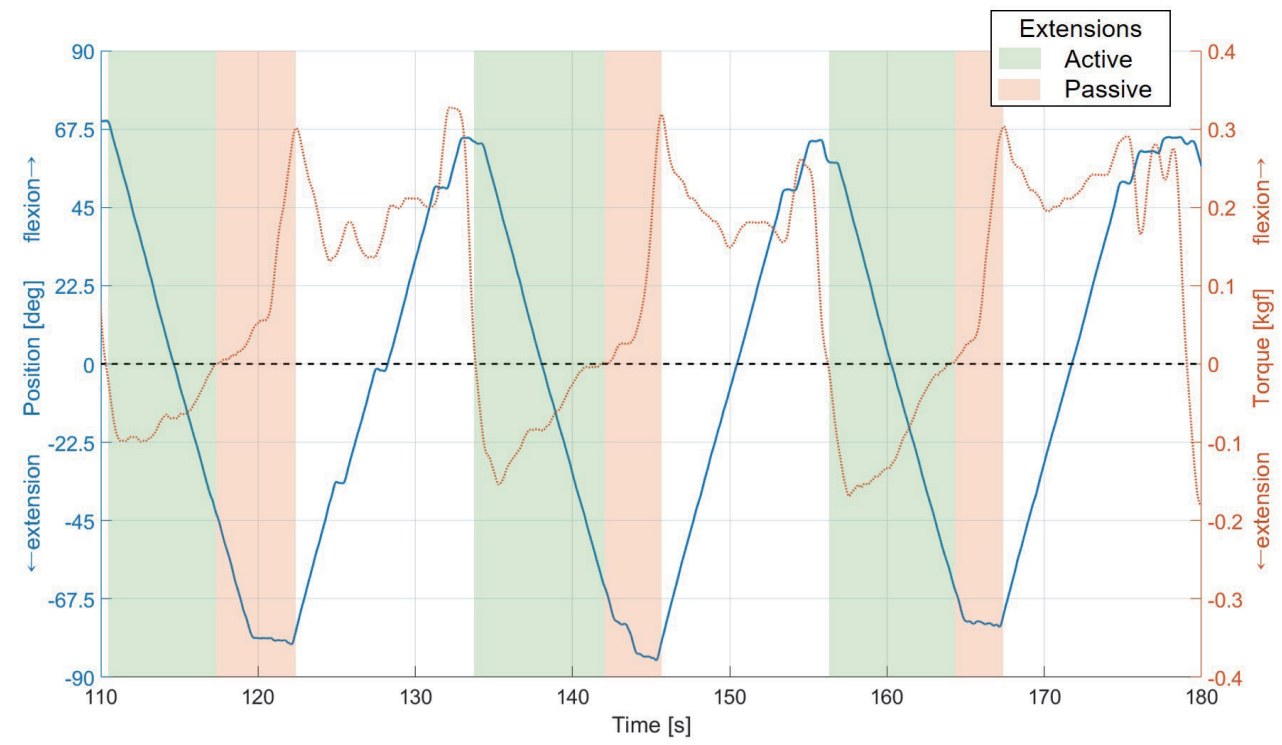

Fig. 6. (Color online) Wrist position and torque of a patient at Brunnstrom stage 4 during the flexion-extension exercise from 110 to $180 \mathrm{~s}$ out of $10 \mathrm{~min}$. During extension, the sign of the torque changes from negative to positive as reflexive characteristics appear at the extreme extension position.

attention, prompting us to analyze our data more thoroughly. We soon discovered that patients at Brunnstrom stages 4 and 5 actively performed the wrist extension exercise with their own wrist extension torque. However, at extreme angles at the extension position, the direction of the torque data changed from extension to flexion, as shown in Fig. 6.

We interpreted the borders between the shaded regions as transitions from active to passive extension. In the field of rehabilitation, the combination of active and passive extension exercises is called an active-assistive method and is considered to be one of the most effective methods for stimulating neuroplasticity. In many other studies, viscosity and stiffness were also used as joint kinematic parameters for poststroke patients, but only for passive exercises. In our study, the active-assistive exercises with the mirroring robot allowed us to calculate the viscosity coefficient $B$ with negative values for subjects at Brunnstrom stages 4 and 5 . Owing to the expansion of the variable space, the machine learning results in Figs. 4 and 5 were reliable and intuitively understandable. We expect that consistent outcomes will be obtained from planned joint studies with rehabilitation experts at hospitals and human motor control professionals at international research institutions.

\section{Conclusions}

In this paper, using a rehabilitation robot we developed, we quantitatively evaluated the severity of wrist paralysis in stroke hemiplegic patients. Stiffness and viscosity constants were estimated on the basis of the torque, angle, and angular velocity vectors of the paralyzed wrist measured by the robot sensors during 10 minutes of flexion-extension exercises. The results showed the high correlation between these constants. Through machine learning, the two parameters were found to be in accord with the Brunnstrom stages of the patients, and musculoskeletal paralysis could be more finely analyzed within the same stage. 


\section{Acknowledgments}

This work was supported by a grant from the National Research Foundation of Korea (NRF) funded by the Korea government (MOE) (No. 2017R1D1A3B03035973).

\section{References}

1 Z. Corbyn: Nature $\mathbf{5 1 0}$ (2014) S2.

2 World Health Organization: Global status report on noncommunicable diseases 2014. No. WHO/NMH/ NVI/15.1. World Health Organization, 2014.

3 M. L. Niemi, R. Laaksonen, M. Kotila, and O. Waltimo: Stroke 19 (1988) 1101.

4 R. B. King: Stroke 27 (1996) 1467.

5 W. M. Hopman and J. Verner: Stroke 34 (2003) 801.

6 Stroke Association: https://www.stroke.org.uk/sites/default/files/stroke_statistics_2015.pdf (accessed September 2019).

7 B. B. Johansson: Stroke 31 (2000) 223.

8 A. Brashear, M. F. Gordon, E. Elovic, V. D. Kassicieh, C. Marciniak, M. Do, C. H. Lee, S. Jenkins, and C. Turkel: N. Engl. J. Med. 347 (2002) 395.

9 G. V. Smith, K. H. Silver, A. P. Goldberg, and R. F. Macko: Stroke 30 (1999) 2112.

10 P. Langhorne, F. Coupar, and A. Pollock: Lancet Neurol. 8 (2009) 741.

11 J. W. Jutai and R. W. Teasell: Stroke Rehabil. 10 (2003) 71.

12 J. Kim and J. Kim: Proc. 39th Ann. Int. Conf. IEEE Engineering in Medicine and Biology Society (EMBC) (IEEE, 2017) p. 4243.

13 E. De Vlugt, J. H. de Groot, K. E. Schenkeveld, J. Arendzen, F. C. van Der Helm, and C. G. Meskers: J. NeuroEng. Rehabil. 7 (2010) 35.

14 M. M. Mirbagheri, L. Alibiglou, M. Thajchayapong, and W. Z. Rymer: J. NeuroEng. Rehabil. 5 (2008) 6.

15 D. Piovesan, P. Morasso, P. Giannoni, and M. Casadio: IEEE Trans. Neural Syst. Rehabil. Eng. 21 (2012) 454.

16 C. G. Meskers, A. C. Schouten, J. H. de Groot, E. de Vlugt, B. J. van Hilten, F. C. Van der Helm, and H. J. Arendzen: J. NeuroEng. Rehabil. 6 (2009) 29.

17 J. Lee, Y. Kagamihara, and S. Kakei: PLoS One 10 (2015) e0132983.

\section{About the Authors}

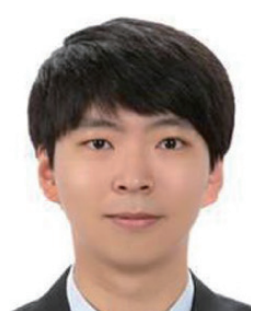

Jihun Kim received his M.S. degree from Handong Global University, South Korea in 2017 and is currently pursuing his Ph.D. degree in mechanical and control engineering at Handong Global University, South Korea. As a researcher in Human Robotics Laboratory, he studies human motor control, biosignals, and robot control. In his current research, he aims to develop rehabilitation robots for stroke hemiplegic patients.

(21738003@ handong.edu)

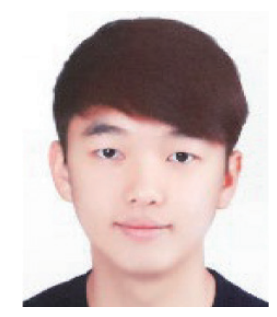

Wookhyun Park received his B.S. degree from Handong Global University, South Korea in 2018 and is currently pursuing his M.S. degree in mechanical and control engineering at Handong Global University, South Korea. As a researcher in Human Robotics Laboratory, his research interests are in bioengineering and robotics. (whpark.mce@gmail.com) 


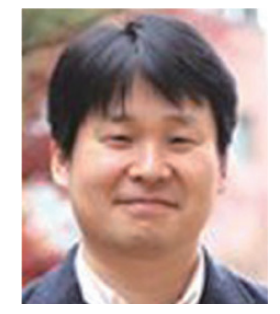

Jaehyo Kim received his Ph.D. degree from Tokyo Institute of Technology, Japan, and is a professor at the School of Mechanical and Control Engineering at Handong Global University, South Korea. He is an academic director of the Korean Society of Mechanical Engineers. His studies include computational neuroscience and human interface. His current research is mostly focused on the development of rehabilitation systems for stroke and neurological patients. (jhkim@handong.edu) 